February 6, 2020

\title{
ON ZEROS OF EXPONENTIAL POLYNOMIALS AND QUANTUM ALGORITHMS
}

\author{
YOSHITAKA SASAKI*
}

\begin{abstract}
We calculate the zeros of an exponential polynomial of some variables by a classical algorithm and quantum algorithms which are based on the method of van Dam and Shparlinski, they treated the case of two variables, and compare with the complexity of those cases. Further we consider the ratio (classical/quantum) of the complexity. Then we can observe the ratio is virtually 2 when the number of the variables is sufficiently large.
\end{abstract}

\section{INTRODUCTION}

For a prime number $p$, we put $q=p^{\nu}$, where $\nu$ is a certain positive integer. Then we denote the finite field by $\mathbb{F}_{q}$ which has $q$ elements. Namely, $\mathbb{F}_{q}$ forms an additive group and $\mathbb{F}_{q}^{\times}:=\mathbb{F}_{q} \backslash\{0\}$ forms a multiplicative group, where 0 is the zero element in $\mathbb{F}_{q}$. Any element of $\alpha \in \mathbb{F}_{q}^{\times}$have a periodicity, that is there exists a smallest natural number $s$ such that $\alpha^{s}=1$. We call such $s$ the "multiplicative order" of $\alpha$. It is known that the multiplicative order is a divisor of $\# \mathbb{F}_{q}^{\times}=q-1$. See [8], [2] for the details.

To evaluate the number of zeros of a homogeneous polynomial

$$
F\left(x_{0}, \ldots, x_{m}\right)=\sum_{\left(n_{0}, \ldots, n_{m}\right) \in \mathbb{N}_{0}^{m+1}} a_{n_{0}, \ldots, n_{m}} x_{0}^{n_{0}} \cdots x_{m}^{n_{m}}
$$

is a very important problem in mathematics. Here, $\mathbb{N}_{0}:=\mathbb{N} \cup\{0\}$ and $a_{n_{1}, \ldots, n_{m}} \in \mathbb{F}_{q}$. The zeta-function associated with such polynomial (the congruence zeta-function) was introduced to treat this problem. Particularly, the zeros of the congruence zeta-function satisfies an analogue of the Riemann hypothesis called "Weil conjecture". Therefore to compute the zeros of the congruence zeta-function is very important investigation. In [4], van Dam studied the zeros of the zeta-function associated with the Fermat surface by using quantum computing.

In [5], van Dam and Shparlinski treated the following exponential polynomial

$$
f(x, y)=a_{1} g_{1}^{x}+a_{2} g_{2}^{y}-b
$$

Key words and phrases. Quantum computing, Exponential congruence, Discrete logarithm, Character sum.

*Partly supported by "Open Research Center" Project for Private Universities: matching fund subsidy from MEXT. 
and calculated the zeros of (1.1) by quantum algorithms. Further they compared the complexity due to a classical algorithm with that due to a quantum algorithm. Then the "cubic" speed-up was observed.

In this article, we treat the exponential polynomial of $n$ variables

$$
f_{b}\left(x_{1}, \ldots, x_{n}\right):=a_{1} g_{1}^{x_{1}}+\cdots+a_{n} g_{n}^{x_{n}}-b
$$

We restrict $n \ll q^{\varepsilon}$ with a small $\varepsilon>0$. The reason why we claim this restriction will be explained in Appendix, below. We calculate the solutions of $f_{b}\left(x_{1}, \ldots, x_{n}\right)=0$ by using quantum algorithms which are natural generalizations of the method of van Dam and Shparlinski. Here, $a_{i}, g_{i} \in \mathbb{F}_{q}^{\times}(i=1, \ldots, n)$ and $b \in \mathbb{F}_{q}$. Further we also compare the complexity due to a classical algorithm with that due to a quantum algorithm. Then exponentially " $(2 n-1) /(n-1)$ " times speed-up is observed. We notice that $(2 n-1) /(n-$ $1)=2+1 /(n-1)$ is virtually 2 when $n$ is sufficiently large. This is the boundary between a standard classical algorithm and our quantum algorithm. In the previous paper [9], Ohno, the author and Yamazaki treated the case of three variables and obtained the ratio $5 / 2$.

In the next section, we introduce some notation and give the considerable lemma which supports whether there exist the zeros of (1.2). In Section 3, we evaluate the complexity due to a classical algorithm. Further in Section 4, we evaluate the complexity due to a quantum algorithm.

\section{THE NUMBER OF SOLUTION OF EQUATION}

In this section, we give an important formula with respect to the density of solutions of

$$
f_{b}\left(x_{1}, \ldots, x_{n}\right)=0
$$

as Lemma 2.1, below. To state it, we introduce some notation.

Let each $s_{i}$ be the multiplicative order of $g_{i}(i=1, \ldots, n)$ in $(2.1)$. We put

$$
\begin{aligned}
X_{i} & :=\left\{0,1, \ldots, s_{i}-1\right\} \cong \mathbb{Z} / s_{i} \mathbb{Z}, \quad(i=1, \ldots, n), \\
X_{n}(r) & :=\{0,1, \ldots, r-1\} \subseteq X_{n} \quad\left(r=1,2, \ldots, s_{n}\right), \\
\boldsymbol{X}^{n}(r) & :=X_{1} \times \cdots \times X_{n-1} \times X_{n}(r), \\
\boldsymbol{X}^{n} & :=\boldsymbol{X}^{n}\left(s_{n}\right)=X_{1} \times \cdots \times X_{n-1} \times X_{n}
\end{aligned}
$$

and

$$
\vec{x}:=\left(x_{1}, \ldots, x_{n}\right) \in \boldsymbol{X}^{n}(r) .
$$


Then we define

$$
\begin{aligned}
S_{f_{b}}(r) & :=\left\{\left(x_{1}, \ldots, x_{n}\right) \in \boldsymbol{X}^{n}(r) \mid f_{b}\left(x_{1}, \ldots, x_{n}\right)=0\right\} \\
N_{f_{b}}(r) & :=\# S_{f_{b}}(r)
\end{aligned}
$$

for $r=1, \ldots, s_{n}$.

By using above notation, we can state the following result:

Lemma 2.1. Let $\delta$ be a parameter satisfying $\delta=o(q)$. For $r>\delta^{2} q^{n}\left(\prod_{l=1}^{n-1} s_{l}\right)^{-2}$, we have

$$
N_{f_{b}}(r)=\frac{r \prod_{l=1}^{n-1} s_{l}}{q}+O\left(\delta \sqrt{r q^{n-2}}\right),
$$

except for at most $q / \delta^{2}$ exceptional b's. Further $O$-constant can be taken 1.

Choosing $\delta=(\log q)^{1 / 2}$ in Lemma 2.1, we have

Corollary 2.2. If $q^{n}\left(\prod_{l=1}^{n-1} s_{l}\right)^{-2} \log q<r \leq s_{n}$, then we see that $S_{f_{b}}(r) \neq \phi$ holds except for at most $q / \log q$ exceptional b's.

Remark 2.3. The exponent $1 / 2$ of $\delta=(\log q)^{1 / 2}$ is not necessary. In fact, $\delta=(\log q)^{\varepsilon}$ with any $\varepsilon>0$ is sufficient.

Proof of Lemma 2.1. Let $\psi$ be a non-trivial additive character over $\mathbb{F}_{q}$, in fact, any additive character over $\mathbb{F}_{q}$ can be given as a map $\mathbb{F}_{q} \rightarrow \mathbb{C}_{1}^{*}$, where $\mathbb{C}_{1}^{*}:=\{z \in \mathbb{C}|| z \mid=1\}$ (see [8, Theorem 5.7]). To evaluate $N_{f_{b}}(\boldsymbol{v})$, we use the following formula which plays as a counting function:

$$
\frac{1}{q} \sum_{\mu \in \mathbb{F}_{q}} \psi(u \mu)= \begin{cases}1 & \text { if } u=0 \\ 0 & \text { otherwise. }\end{cases}
$$

Then we have

$$
\begin{aligned}
N_{f_{b}}(r) & =\sum_{\vec{x} \in \boldsymbol{X}^{n}(r)} \frac{1}{q} \sum_{\mu \in \mathbb{F}_{q}} \psi\left(\mu\left(f_{b}\left(x_{1}, \ldots, x_{n}\right)\right)\right) \\
& =\frac{r \prod_{j=1}^{n-1} s_{l}}{q}+\frac{1}{q} \sum_{\mu \in \mathbb{F}_{q}^{*}} \sum_{\vec{x} \in \boldsymbol{X}^{n}(r)} \psi\left(\mu\left(f_{b}\left(x_{1}, \ldots, x_{n}\right)\right)\right) \\
& =: \frac{r \prod_{l=1}^{n-1} s_{l}}{q}+\Delta_{b}(r) .
\end{aligned}
$$

If the contribution from the second term on the right-hand side of the above formula can be estimated by $o\left(r \prod_{l=1}^{n-1} s_{l} / q\right)$, the above formula tells us the existence of the solution of $f_{b}\left(x_{1}, \ldots, x_{n}\right)$. To consider it, we evaluate the mean value of the second term on the right-hand side of (2.4) with respect to $b$. Namely, we evaluate

$$
E(r):=\sum_{b \in \mathbb{F}_{q}}\left|\Delta_{b}(r)\right|^{2}
$$


From (2.3) and some properties of the additive character over $\mathbb{F}_{q}$, we obtain

$$
\begin{aligned}
E(r)= & \frac{1}{q^{2}} \sum_{\mu, \mu^{\prime} \in \mathbb{F}_{q}^{\times}}\left(\prod_{j=1}^{n-1}\left(\sum_{x_{j}, x_{j}^{\prime} \in X_{j}} \psi\left(a_{j}\left(\mu g_{j}^{x_{j}}-\mu^{\prime} g_{j}^{x_{j}^{\prime}}\right)\right)\right)\right) \sum_{x_{n}, x_{n}^{\prime} \in X_{n}(r)} \psi\left(a_{n}\left(\mu g_{n}^{x_{n}}-\mu^{\prime} g_{n}^{x_{n}^{\prime}}\right)\right) \\
& \times \sum_{b \in \mathbb{F}_{q}} \psi\left(b\left(\mu^{\prime}-\mu\right)\right) \\
= & \frac{1}{q} \sum_{\mu \in \mathbb{F}_{q}^{\times}}\left(\prod_{j=1}^{n-1}\left(\sum_{x_{j}, x_{j}^{\prime} \in X_{j}} \psi\left(a_{j} \mu\left(g_{j}^{x_{j}}-g_{j}^{x_{j}^{\prime}}\right)\right)\right)\right) \sum_{x_{n}, x_{n}^{\prime} \in X_{n}(r)} \psi\left(a_{n} \mu\left(g_{n}^{x_{n}}-g_{n}^{x_{n}^{\prime}}\right)\right) \\
= & \frac{1}{q} \sum_{\mu \in \mathbb{F}_{q}^{\times}}\left(\prod_{j=1}^{n-1}\left|\sum_{x_{j} \in X_{j}} \psi\left(a_{j} \mu g_{j}^{x_{j}}\right)\right|^{2}\right)\left|\sum_{x_{n} \in X_{n}(r)} \psi\left(a_{n} \mu g_{n}^{x_{n}}\right)\right|^{2} .
\end{aligned}
$$

It is known that

$$
\left|\sum_{x_{j} \in X_{j}} \psi\left(a_{j} \mu g_{j}^{x_{j}}\right)\right| \leq \sqrt{q} \text { for } j=1, \ldots, n-1 \text { and any } \mu \in \mathbb{F}_{q}^{\times}
$$

(see Theorem 8.78 in [8]). Hence we have

$$
E(r)<q^{n-2} \sum_{\mu \in \mathbb{F}_{q}}\left|\sum_{x_{n} \in X_{n}(r)} \psi\left(a_{n} \mu f^{x_{n}}\right)\right|^{2}=q^{n-1} r .
$$

Therefore, if we put $\delta=o(q)$, then we can see that there exist at most $q / \delta^{2}$ exceptional b's such that

$$
\left|\frac{1}{q} \sum_{\mu \in \mathbb{F}_{q}^{*}} \sum_{\vec{x} \in X_{n}(r)} \psi\left(\mu\left(f_{b}\left(x_{1}, \ldots, x_{n}\right)\right)\right)\right| \geq \delta \sqrt{r q^{n-2}}
$$

Hence we obtain

$$
N_{f_{b}}(r)=\frac{r \prod_{l=1}^{n-1} s_{l}}{q}+O\left(\delta \sqrt{q^{n-2} r}\right)
$$

for other b's. Now, the proof of Lemma 2.1 is completed.

\section{Calculation of the Deterministic time for a Classical Algorithm}

We follow the method of van Dam and Shparlinski [5]. Then we have

Theorem 3.1. Except for at most $q / \log q$ exceptional b's, we can either find a solution $\vec{x} \in \boldsymbol{X}^{n}$ of the equation (2.1) or decide that it does not have a solution in deterministic time $q^{n(n+1) / 2(2 n-1)}(\log q)^{O(1)}$ as a classical computer. 
Proof. Using a standard deterministic factorization algorithm, we factorize $q-1$ and find the orders $s_{j}$ of $g_{j}(j=1, \ldots, n)$ in time $q^{1 / 2}(\log q)^{O(1)}$. We may assume without loss of generality that $s_{1} \geq \cdots \geq s_{n}$. For calculated orders $s_{1}, \ldots, s_{n-1}$, we put

$$
r=\left\lceil q^{n}\left(\prod_{l=1}^{n-1} s_{l}\right)^{-2} \log q\right\rceil .
$$

Then we see that the solution of (2.1) certainly exists when $r \leq s_{n}$. However, when $r>s_{n}$, we do not know whether such solutions exist. Therefore we have to consider those two cases.

For each $\left(x_{2}, \ldots, x_{n-1}, x_{n}\right) \in X_{2} \times \cdots \times X_{n-1} \times X_{n}(r)$, we calculate the deterministic time of the discrete logarithm $x_{1}$ such that $g_{1}^{x_{1}}=a_{1}^{-1}\left(b-a_{2} g_{2}^{x_{2}}-\cdots-a_{n} g_{n}^{x_{n}}\right)$. It is known that the deterministic time for this case is $s_{1}^{1 / 2}(\log q)^{O(1)}$ (see Section 5.3 in [3]).

(i) The case $r \leq s_{n}$. We have

$$
s_{1}^{1 / 2}\left(\prod_{l=2}^{n-1} s_{l}\right) r(\log q)^{O(1)} \ll q^{n / 2}(\log q)^{O(1)},
$$

since $s_{1}^{1 / 2}\left(\prod_{l=2}^{n-1} s_{l}\right) r<\left(\left(\prod_{l=1}^{n-1} s_{l}\right)^{2} r\right)^{1 / 2}$.

(ii) The case $r>s_{n}$. Similarly, we see that the deterministic time is

$$
s_{1}^{1 / 2}\left(\prod_{l=2}^{n} s_{l}\right)(\log q)^{O(1)} \ll q^{n / 2}(\log q)^{O(1)},
$$

since $s_{1}^{1 / 2} \prod_{l=2}^{n} s_{l}<\left(\left(\prod_{l=1}^{n-1} s_{l}\right)^{2} s_{n}\right)^{1 / 2}<\left(\left(\prod_{l=1}^{n-1} s_{l}\right)^{2} r\right)^{1 / 2}$.

\section{Calculation of the COMplexity for a QUantum Algorithm}

In this section, we describe quantum algorithms which are based on the method of [5]. Hereafter $\varepsilon$ is any positive and small real number.

Theorem 4.1. Except for at most $q / \log q$ exceptional b's, we can either find a solution $\vec{x} \in \boldsymbol{X}^{n}$ of the equation (2.1) or decide that it does not have a solution in time $q^{n(n-1) / 2(2 n-1)+\varepsilon}(\log q)^{O(1)}$ as a quantum computer.

Proof. Using Shor's algorithm [10], we can obtain the multiplicative orders $s_{j}$ 's $(j=$ $1, \ldots, n)$ in polynomial time. We may assume without loss of generality that $s_{1} \geq \cdots \geq s_{n}$. As in the proof of Theorem 3.1, we put $r$ as (3.1). Further, we consider a polynomial time quantum subroutine $\mathcal{S}\left(x_{2}, \ldots, x_{n}\right)$ which either finds and returns $x_{1} \in X_{1}$ with

$$
g_{1}^{x_{1}}=a_{1}^{-1}\left(b-a_{2} g_{2}^{x_{2}}-\cdots-a_{n} g_{n}^{x_{n}}\right)
$$

or reports that no such $x_{1}$ exists for a given $\left(x_{2}, \ldots, x_{n-1}, x_{n}\right) \in X_{2} \times \cdots \times X_{n-1} \times X_{n}(r)$ by using Shor's discrete logarithm algorithm. 
(i) The case $r \leq s_{n}$. Using Grover's search algorithm [6], we search the subroutine $\mathcal{S}\left(x_{2}, \ldots, x_{n}\right)$ for all $\left(x_{2}, \ldots, x_{n-1}, x_{n}\right) \in X_{2} \times \cdots \times X_{n-1} \times X_{n}(r)$ in time

$$
q^{\varepsilon}\left(r \prod_{l=2}^{n-1} s_{l}\right)^{1 / 2}(\log q)^{O(1)} \ll q^{n(n-1) / 2(2 n-1)+\varepsilon}(\log q)^{O(1)},
$$

since $r \prod_{l=2} s_{l} \leq\left(\left(\prod_{l=1}^{n-1} s_{l}\right)^{2} r\right)^{(n-1) /(2 n-1)}$.

(ii) The case $r>s_{n}$. Similarly, we search the $\mathcal{S}\left(x_{2}, \ldots, x_{n}\right)$ for all $\left(x_{2}, \ldots, x_{n-1}, x_{n}\right) \in$ $X_{2} \times \cdots \times X_{n-1} \times X_{n}(r)$ in time

$$
\begin{gathered}
q^{\varepsilon}\left(\prod_{l=2}^{n} s_{l}\right)^{1 / 2}(\log q)^{O(1)} \ll q^{n(n-1) / 2(2 n-1)+\varepsilon}(\log q)^{O(1)}, \\
\text { since } \prod_{l=2}^{n} s_{n} \leq\left(\left(\prod_{l=1}^{n-1} s_{l}\right)^{2} s_{n}\right)^{(n-1) /(2 n-1)}<\left(\left(\prod_{l=1}^{n-1} s_{l}\right)^{2} r\right)^{(n-1) /(2 n-1)} .
\end{gathered}
$$

In [5], van Dam and Shparlinski mentioned when the multiplicative orders are large, there is a more efficient quantum algorithm. Similarly, we can also consider a more efficient quantum algorithm.

Theorem 4.2. If we assume

$$
\left(\prod_{l=1}^{n-1} s_{l}\right)^{2} s_{n}>q^{n} \log q
$$

then we can either find a solution $\vec{x} \in \boldsymbol{X}^{n}$ of the equation (2.1) or decide that it does not have a solution in time $q^{1 / 2+\varepsilon}\left(\left(\prod_{l=1}^{n-1} s_{l}\right)^{2} s_{n}\right)^{-1 / 2(2 n-1)}(\log q)^{O(1)}$ as a quantum computer, except for at most $q / \log q$ exceptional b's.

Remark 4.3. The upper bound of the running time of the algorithm of Theorem 4.2 is

$$
O\left(q^{(n-1) / 2(2 n-1)+\varepsilon}(\log q)^{O(1)}\right) .
$$

Proof of TheOrem 4.2. We may assume without loss of generality that $s_{1} \geq s_{2} \geq$ $s_{3}$. We put

$$
r=\left\lfloor q^{n}\left(\prod_{l=1}^{n-1} s_{l}\right)^{-2} \log q\right\rfloor
$$

Then from the assumption of the theorem we see that $r \leq s_{n}$. Hence there are some solutions of (2.1) in $\boldsymbol{X}^{n}(r)$ and we denote the number of the solutions of (2.1) by $M$. Note that $M \asymp\left(r \prod_{l=1}^{n-1} s_{l}\right) / q$.

As in the case of [5], we use the version of Grover's algorithm as described in [1] that finds one out of $m$ matching items in a set of size $t$ by using only $O(\sqrt{t / m})$ queries. We 
search the subroutine $\mathcal{S}\left(x_{2}, \ldots, x_{n}\right)$ for all $\left(x_{2}, \ldots, x_{n-1}, x_{n}\right) \in X_{2} \times \cdots \times X_{n-1} \times X_{n}(r)$. Then the complexity is

$$
q^{\varepsilon}\left(\frac{\left(\prod_{l=2}^{n-1} s_{l}\right) r}{M}\right)^{1 / 2}(\log q)^{O(1)} \leq q^{1 / 2+\varepsilon}\left(\left(\prod_{l=1}^{n-1} s_{l}\right)^{2} s_{n}\right)^{-1 / 2(2 n-1)}(\log q)^{O(1)}
$$

\section{Concluding Remarks}

See the following list.

\begin{tabular}{|c|c|c|c|}
\hline \# of variables & Classical & Quantum & ratio $(\mathrm{C} / \mathrm{Q})$ \\
\hline 2 (van Dam and Shparlinski) & 1 & $1 / 3$ & 3 \\
\hline 3 (Ohno, S, Yamazaki) & $3 / 2$ & $3 / 5$ & $5 / 2$ \\
\hline$\vdots$ & $\vdots$ & $\vdots$ & $\vdots$ \\
\hline$n$ & $n / 2$ & $n(n-1) / 2(2 n-1)$ & $(2 n-1) /(n-1)$ \\
\hline
\end{tabular}

We notice that the ratio is virtually 2 when $n$ is sufficiently large. It seems to come from the effect of Grover's algorithm.

\section{REFERENCES}

[1] M. Boyer, G. Brassard, P. Høyer And A. TApp, Tight bounds on quantum searching, Fortschritte der Physik, 46 (1998), 493-505.

[2] A. M. Childs And W. VAn DAm, Quantum algorithms for algebraic problems, Reviews of Modern Physics, to appear.

[3] R. Crandall and C. Pomerance, Prime Numbers: A computational perspective, Springer-Verlag, Berlin, 2005.

[4] W. VAN DAM, Quantum computing and zeroes of zeta functions, preprint, arXiv:0405081[quant-ph].

[5] W. van Dam and E. ShParlinski, Classical and Quantum Algorithms for Exponential Congruences, Proceedings of the Third Workshop on Theory of Quantum Computation, Communication and Cryptography (TQC 2008), Lecture Notes in Computer Science, Vol. 5106, 1-10 (2008)

[6] L. Grover, A fast quantum-mechanical algorithm for database search, Proceedings of the 28th Annual ACM Symposium on Theory of Computing (STOC '96), 1996, pp. $212-219$.

[7] A. Ivić, The Riemann Zeta-Function, Wiley, New York, 1985.

[8] R. Lidl And H. Niederreiter, Finite Fields, Encyclopedia of Mathematics and Its Applications, Vol. 20, Cambridge Univ. Press, Cambridge, 1997.

[9] Y. Ohno, Y. Sasaki And C. Yamazaki, On exponential polynomials and quantum computing, preprint, arXiv:0908.1027[quant-ph]. 
[10] P. Shor, Polynomial-time algorithms for prime factorization and discrete logarithms on a quantum computer, SIAM Journal on Computing 26 (1997), 1484-1509.

\section{ApPEndix A. ApPEndix}

For a natural number $n$, we define the divisor function by

$$
d(n):=\#\{d \in \mathbb{N}|d| n\}=\sum_{d \mid n} 1 .
$$

In Section 1, we introduced the notion of the multiplicative order $s$ of $g \in \mathbb{F}_{q}^{\times}$and mentioned the multiplicative order is a divisor of $q-1$.

We put $g_{1}$ and $g_{2}$ have the same multiplicative order. Then there exists a natural number $l$ such that $g_{2}=g_{1}^{l}$. Hence, we have

$$
a_{1} g_{1}^{x_{1}}+a_{2} g_{2}^{x_{2}}=a_{1} g_{1}^{x_{1}}+a_{2} g_{1}^{l x_{2}},
$$

where $a_{i}, g_{i} \in \mathbb{F}_{q}^{\times}(i=1,2)$. The right-hand side of the above equation is a element of $\overline{a_{1}}+\overline{a_{2}} \in \mathbb{F}_{q}^{\times} /\left\langle g_{1}\right\rangle$, where $\bar{a}$ is a coset of $\mathbb{F}_{q}^{\times} /\left\langle g_{1}\right\rangle$ and $\left\langle g_{1}\right\rangle$ is the cyclic group generated by $g_{1}$. Therefore our central problem (1.2) is reduced to

$$
\widetilde{f}_{b}\left(z_{1}, \ldots, z_{\mu}\right)=c_{1} h_{1}^{z_{1}}+\cdots+c_{\mu} h_{\mu}^{z_{\mu}}-b=0,
$$

where each $h_{i}(i=1, \ldots, \mu)$ does not have the same multiplicative order and $\mu \leq d(q-1)$.

It is known that

$$
d(n) \ll n^{\varepsilon}
$$

for any positive number $\varepsilon$ (for instance, see [7]). Hence, we have

$$
\mu \ll q^{\varepsilon}
$$

for any $\varepsilon>0$.

Interdisciplinary Graduate School of Science and Engineering

KinKI UNIVERSITY

Higashi-Osaka, Osaka 577-8502

JAPAN

E-mail address: sasaki@alice.math.kindai.ac.jp 